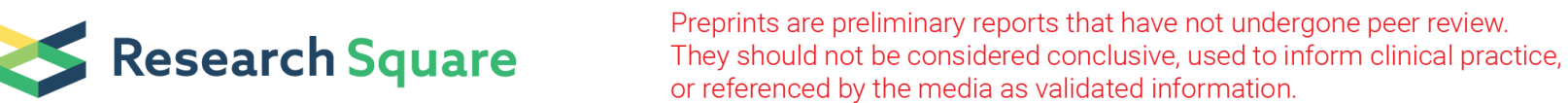

\section{SARS-CoV-2 specific B cell memory drives improved class switching and tissue homing responses to single dose Ad26.COV2.S vaccination in previously infected recipients}

\section{Rob Krause}

Africa Health Research Institute

Thandeka Moyo-Gwete

MRC Antibody Immunity Research Unit

\section{Simone Richardson}

National Institute of Communicable Diseases https://orcid.org/0000-0001-7678-2609

\section{Zanele Makhado}

MRC Antibody Immunity Research Unit

Nelia Manamela

South African Medical Research Council Antibody Immunity Research Unit, Faculty of Health Sciences, University of the Witwatersrand, Johannesburg, South Africa

\section{Tandile Hermanus}

MRC Antibody Immunity Research Unit

\section{Nono Mkhize}

National Institute for Communicable Diseases

\section{Roanne Keeton}

Division of Medical Virology

Ntombi Benede

Division of Medical Virology

Mathilda Mennen

Department of Medicine

\section{Sango Skelem}

Department of Medicine

\section{Catherine Riou}

University of Cape Town https://orcid.org/0000-0001-9679-0745

\section{Ntobeko Ntusi}

University of Cape Town https://orcid.org/0000-0003-2347-7883

\section{Ameena Goga}

Clinical Trials Unit

\section{Glenda Gray}


South African Medical Research Council

\section{Nigel Garrett}

Centre for the AIDS Program of Research in South Africa (CAPRISA), University of KwaZulu-Natal, Durban, South Africa 4041 https://orcid.org/0000-0002-4530-234X

\section{Linda-Gail Bekker}

Desmond Tutu HIV Centre

\section{Andreas Groll}

Department of Statistics https://orcid.org/0000-0001-6787-9118

\section{Penny Moore}

National Institute of Communicable Diseases https://orcid.org/0000-0001-8719-4028

\section{Wendy Burgers}

Division of Medical Virology

\section{Alasdair Leslie ( $\sim$ al.leslie@ahri.org )}

Africa Health Research Institute https://orcid.org/0000-0003-2538-6467

\section{Article}

\section{Keywords:}

Posted Date: January 12th, 2022

DOI: https://doi.org/10.21203/rs.3.rs-1170883/v1

License: (c) (i) This work is licensed under a Creative Commons Attribution 4.0 International License. Read Full License 


\section{Abstract}

Neutralizing antibodies strongly correlate with protection for COVID-19 vaccines, but the corresponding memory $B$ cells that form to protect against future infection are relatively understudied. Here we examine the effect of prior SARS-CoV-2 infection on the magnitude and phenotype of the B cell response to single dose Johnson and Johnson (Ad26.COV2.S) vaccination in South African health care workers. SARS-CoV2 specific memory responses expand in response to Ad26.COV2.S and are maintained for the study duration (84 days) in all individuals. However, prior infection is associated with a greater frequency of these cells, a more prominent germinal center (GC) response, and increased class switched memory (CSM). These B cell features correlated with both neutralization and antibody-dependent cytotoxicity (ADCC) activity, and with the frequency of SARS-CoV-2 specific circulating T follicular helper cells (cTfh). In addition, the SARS-CoV-2 specific CD8+ T cell response correlated with increased memory B cell lunghoming, which was sustained in the infected group. Finally, although vaccination achieved equivalent $B$ cell activation regardless of infection history, it was negatively impacted by age. These data show that phenotyping the $\mathrm{B}$ cell response to vaccination can provide mechanistic insight into the impact of prior infection on GC homing, CSM, cTfh, and neutralization activity. These data can provide early signals and mechanistic understanding to inform studies of vaccine boosting, durability, and co-morbidities.

\section{Introduction}

The COVID-19 pandemic has caused more than 269 million infections and 5.3 million deaths (WHO dashboard: 14 Dec 2021). As global vaccination programs progress, vaccines are being administered to individuals with or without prior exposure to COVID-19. Therefore, it is important to understand the dynamics of the vaccine response in these two groups, as this could inform future vaccine dosing, scheduling and provide insight into homogeneous or heterogeneous vaccine boosting. In addition, it provides a unique opportunity to study immunological mechanisms that govern vaccine effectiveness and longevity and uncover potential early biomarkers of these essential metrics. All currently approved COVID-19 vaccines are based on the ancestral SARS-CoV-2 Wuhan-1 viral spike protein ${ }^{1-3}$, which facilitates viral entry into host cells via the host ACE2 and TMPRS22 surface receptors ${ }^{4}$. Eliciting antibodies against this spike and its receptor binding domain (RBD) can block viral adhesion and neutralize it, thereby preventing infection ${ }^{5-9}$. A functional antibody response therefore serves as an important correlate of protection for vaccine efficacy ${ }^{10,11}$ and includes Fc-dependent antibody effector functions ${ }^{12}$. However, as SARS-CoV-2 variants continue to emerge, the specificity and cross-reactivity of antibodies elicited through infection, vaccination, or the combination of both remain to be established as their affinity for and functionality against new variants may differ. This is to be expected, since infection would prime responses to the whole virus and would depend on the infecting variant, whereas vaccines generally induce a spike specific response, with most vaccines based on the ancestral virus.

Adenovirus vectored and mRNA vaccines targeting the viral spike have been rolled out worldwide, where the former are considerably cheaper to produce and distribute ${ }^{13}$. Following positive safety and 
immunogenicity data ${ }^{3}$, the single dose Ad26.COV2.S vaccine, manufactured by Johnson and Johnson, was distributed to health care workers as an early response to the pandemic in South Africa (SISONKE trial at clinicaltrials.gov NCT04838795). In this vaccine, the human Adenovirus 26 vector displays a stabilized form of the SARS-CoV-2 spike on its surface ${ }^{1}$. It induces functional antibody and T cell responses against the vaccine strain that although reduced, cross react with several SARS-CoV-2 variants $^{14-16}$. Although the neutralizing antibody response following Ad26.COV2.S vaccination is threeto-five-fold lower than titers triggered by mRNA vaccines ${ }^{14-18}$, it showed an overall effectiveness of $82 \%$ against severe COVID-193.

Functional antibodies, elicited by both infection and vaccination, are produced by antigen specific memory B cells. These long-lived cells are a vital component for protection from future infection and severe disease ${ }^{19,20}$. Infection with SARS-CoV-2 elicits a memory B cell response to COVID-19, which matures five to eight months after infection even though circulating antibodies may wane ${ }^{21-24}$. Studies of individuals receiving an mRNA vaccine found that prior infection profoundly boosted the specific $B$ cell memory response ${ }^{2,25}$. Likewise, prior infection has been shown to boost SARS-CoV-2 specific antibody titers following vaccination ${ }^{16,26-30}$. However, the memory $B$ cell response induced by the single dose Ad26.COV2.S vaccine has not been studied to date, nor has the impact of prior infection.

Here we tested samples collected as part of SISONKE, a phase 3B trial of Ad26.COV.2.S in South African health care workers ${ }^{31}$. COVID-19 seropositivity before vaccination within this sub-cohort was $56 \%{ }^{16}$. We demonstrate that prior infection significantly increases the magnitude of the SARS-CoV-2 specific memory B cell response and its capacity to home to germinal centers and class switch following vaccination. This ultimately boosts the quantity and quality of the antibody response. We show strong associations between several B cell characteristics and quality of the vaccine response, both before and after vaccination, particularly with the degree of germinal center homing and class switching. These were complemented with SARS-CoV-2 specific cTfh and CD8+ responses. Overall, these data provide mechanistic insights into the boosting effects of prior infection on the $B$ cell response to the single dose Johnson and Johnson Ad26.COV2.S vaccination.

\section{Results}

To determine the impact of prior SARS-CoV-2 infection on the frequency and phenotype of the antigen specific memory B cell response to the Johnson and Johnson Ad26.COV2.S vaccine, we studied 30 vaccinated participants, with known prior infection history, sampled before and after receiving a single dose of Ad26.COV2.S. Participants were grouped accordingly: naïve (for those with no history of infection) and infected (for those previously infected with COVID-19). Each participants' COVID-19 history was confirmed by RT-PCR or serology (Roche Elecsys ${ }^{\circledR} S$ and $N$ test) or both. The infected group was further separated into those from the first wave (May to August 2020) when the ancestral D614G virus dominated, the second wave (November 2020 to January 2021) when the Beta variant was dominant, and those for whom no data is available regarding timing of infection or viral sequence (Extended Data 
Table 1). For all participants, peripheral blood mononuclear cells (PBMCs) were obtained prior to vaccination and 28 days post vaccination. As antigen specific memory B cell populations developing in secondary lymphoid organs can be detected in the blood from 21 days after antigen exposure ${ }^{25,32}$. For a subset of participants, samples were also available from day 56 and 84 post vaccination.

SARS-CoV-2 specific B cells were identified using fluorochrome-labeled viral spike and receptor binding domain (RBD) protein. SARS-CoV-2 specific memory B cells were identified by the expression of CD19, CD27 and binding either spike alone (Spike+), or spike and RBD together (Spike+RBD+; Fig. 1a). As expected, both the Spike+ and Spike+RBD+ populations were significantly expanded at day 28 post vaccination (Fig. 1b). Notably, there was a significantly higher Spike+ and Spike+RBD+ memory B cell frequency prior to vaccination (day 0 ) in the infected group from the first wave of the epidemic relative to the naïve group ( $P=0.0006$, data not shown) and the second wave infections $(P=0.018$, data not shown). The frequency of Spike+ and Spike+RBD+ memory B cells post vaccination in naïve individuals was equivalent to the pre-vaccination levels of first wave infected individuals. By contrast, individuals infected in the second wave with the Beta variant had similar pre and post vaccine B cell responses to naïve individuals to spike alone; however, this group had the greatest fold increase of Spike+RBD+ memory B cells (4.5-fold). It is important to note that the baits were made using the D614G ancestral virus proteins, and thus Beta variant spike specific B cells at baseline could have been underestimated. Indeed, based on this frequency data, the cross reactivity of these B cells at baseline appears to have been minimal. In a subset of individuals with additional time points, we confirmed the Spike+ and Spike+RBD+ memory B cell responses persisted for three months in both the naïve and infected groups (Fig. 2a and b); with both showing a non-significant downward trend from day 28 . The median frequency for the infected group was significantly greater than naïve individuals at all time points.

In parallel, we quantified SARS-CoV-2 specific antibodies in matched plasma samples and observed similar trends. As shown in different participants from the same cohort ${ }^{16}$, previous infection significantly boosted the total SARS-CoV-2 D614G and Beta binding antibodies (Fig. 2c and d). In naïve individuals, binding antibody titers also increased but, as with memory $B$ cells, only reached the levels seen prior to vaccination in the infected group. Notably, these data indicate that the Ad26.COV2.S vaccine had a strong boosting effect on individuals previously infected with the Beta variant, despite the apparently minimal $B$ cell cross-reactivity at baseline between first and second wave samples detected using D614G baits.

To determine the relationship between SARS-CoV-2 specific B cells and antibodies prior to vaccination, we correlated Spike+RBD+ memory B cell frequency and binding spike specific antibody titer in baseline samples (Fig. 2e). There was a strong and highly significant correlation between baseline Spike+RBD+ memory B cell frequency and D614G specific antibodies in previously infected individuals $(R=0.84 ; P<$ 0.001), confirming the expected relationship between these two parameters. In addition, although the antibody titers and $B$ cell frequencies were much lower, a similar trend was observed in naïve individuals $(R=0.6 ; P=0.09)$. Although this did not reach statistical significance, it suggests that low frequency spike-cross-reactive B cells exist in individuals who have not been infected with SARS-CoV-2. 
To examine class switching, an important process in the maturation of the antibody response ${ }^{33,34}$, we examined surface expression of IgM and IgD on Spike+RBD+ memory B cells. Both naïve and infected groups displayed increased class switching from day 0 to 84 , consistent with a maturing $B$ cell response. However, at all timepoints, a greater proportion of these cells were fully class switched (IgM- and IgD-) in infected individuals, consistent with prior B cell maturation; while in naïve individuals they expressed more IgM (Fig. 2f), as expected for a primary response to antigen ${ }^{35}$. Unswitched IgD+Spike+RBD+ memory B cells were detectable in subjects with prior infection, suggesting a pool of naïve specific B cells persists in these individuals. Overall, these data confirm that prior SARS-CoV-2 infection had a significant impact on the frequency of virus-specific B cell memory, binding antibody titer and class switching.

Since infection induced a detectable spike specific memory response, we were interested in assessing its impact on activation and homing of these $B$ cells following vaccination. The $B$ cell activation profile was assessed by expression of CD21, which is downregulated upon B cell activation by cognate antigen ${ }^{36-39}$. Both the naïve and infected groups showed significantly increased Spike+ memory B cell activation at day 28 post vaccination, which waned longitudinally (Fig. 3a). Although the initial extent and peak activation levels were greater in the infected compared to the naïve group, this was not significant. However, there was a significant positive correlation between the activation of Spike+ memory B cells and the magnitude of the spike specific antibody response at day 28 (Fig. 3b). In addition, activation of total memory B cells was higher in individuals with prior infection (Fig. 3c), potentially indicating stimulation of additional non-bait reactive memory $B$ cells in this group.

To examine B cell homing, we measured the expression of CXCR5, which is required for migration to germinal centers via CXCL13 40,41 . This revealed a striking reduction in CXCR5 expression at day 28 in the infected group (Fig. 3d), which rebounded at day 56 and 84. This phenomenon was less pronounced and was delayed in naïve individuals, with CXCR5 expression reaching its lowest level at day 56. Overall, there was a significant negative correlation between CXCR5 expression levels on Spike+ memory B cells and antibody titer (Fig. 3e), which was not reflected by differences in CXCR5 expression on memory B cells in general (Fig. 3f).

In addition, we measured the expression levels of CXCR3, a marker known to facilitate homing to inflamed lung mucosae during infection via CXCL9 and CXCL1039,42,43. Prior to vaccination, we observed a striking difference in the expression of this marker on Spike+ memory B cells, which was elevated in approximately half of the individuals with previous infection but not in naïve individuals. Following vaccination, CXCR3 expression increased on Spike+ memory B cells in most individuals with low levels prior to vaccination, but not in individuals with elevated CXCR3 expression at this timepoint (Fig. 3g). The median CXCR3 response for the second wave infected participants had a similar peak response to the naïve group at day 28 , but then retained similar CXCR3 expression to the previously infected group at later time points (Extended Data Fig. 3). Overall, we observed a strong positive correlation between expression levels of CXCR3 and spike specific antibody titer at baseline (Fig. 3h). Again, these differences appear to be limited in antigen specific B cells and are not reflected in the general memory B cell pool 
(Fig. 3i). Taken together, these data indicate that prior infection with SARS-CoV-2 results in phenotypic differences in the spike specific memory B cell response to vaccination.

Having observed changes in B cell activation and chemokine receptor expression, next we assessed the relationship between the vaccine-induced $T$ cell response and memory $B$ cells. T cells play an important role in vaccine protection, and $\mathrm{CD} 4+\mathrm{T}$ follicular helper cells $(\mathrm{Tfh}$ ) play an integral role in the germinal center response by providing help to $B$ cells ${ }^{44}$. Using the activation-induced marker (AIM) assay, we measured the $T$ cell response to a peptide pool covering the full spike protein in 26 participants. The frequencies of spike specific CD8+ and CD4+ T cells were determined using co-expression of CD69 and CD137 (for CD8+) and OX40 and CD137 (for CD4+) 45,46. The circulating T follicular helper (cTfh) population was identified based on the expression of CXCR5 and CD45RA (i.e., CXCR5+CD45RA-), and spike specific cells were defined based on co-expression of OX40 and CD25 (Fig. 4a). Vaccination induced spike specific T cells in both naïve and previously infected individuals (Fig. 4b). We examined the relationship between cTfh, and memory B cell class switching (CSM) after vaccination and found a significant positive correlation between the frequencies of spike specific cTfh and Spike+RBD+ CSM B cells at day 28 (Fig. 4c). Interestingly, we observed a significant correlation at day 28 between AIM+ CD 8+ T cells and the Spike+CXCR3+ memory B cells (Fig. 4d). These data suggest that both CD4+ and CD8+ spike specific responses may influence the $B$ cell response to vaccination.

To determine how the observed difference in B cell phenotype related to functional antibody characteristics, we measured antibody neutralization using a pseudovirus-based assay (Fig. 5a,b) and antibody dependent cellular cytotoxicity (ADCC; Fig. 5c,d) against both the D614G ancestral strain (Fig. $5 a, c)$ and the Beta variant (Fig. 5b,d). As expected, vaccination increased neutralization activity against D614G by day 28 , which was significantly higher in individuals with prior infection. For the Beta variant, however, this was only observed from individuals with prior infection, and neutralization activity in naïve individuals remained low and never reached levels seen in previously infected subjects prior to vaccination. Consistent with our previous finding ${ }^{16}$, vaccination boosted ADCC activity against both viral variants irrespective of previous infection. Both neutralization and ADCC activity, however, were significantly higher against the Beta variant in previously infected individuals compared to naïve individuals at all timepoints.

Using a Spearman correlation analysis, we then examined the relationship between the phenotype of Spike+ memory B cells, SARS-CoV-2 specific antibody titer and functional activity and AIM+ T cells (Fig. $6 a$ ) at baseline and post vaccination (day 28). At baseline, we observed strong positive correlations between both the total frequency of Spike+ memory B cells, degree of class switching, and CXCR3 expression and binding antibody titer and functional activity. The activation state and CXCR5 expression levels do not, however, correlate with antibody functionality at this timepoint. Following vaccination (day 28), the frequency of Spike+ memory B cells and degree of class switching remained correlated with these antibody characteristics, but the correlation with CXCR3 expression was lost. In addition, new associations were detected, including a positive correlation between activated spike specific $B$ cells and both binding titer and neutralization activity; and an inverse correlation between CXCR5 expression and 
binding titer, $A D C C$, and neutralization. On days 56 and 84, positive correlations were retained between spike specific $B$ cells and spike binding antibodies and notably day 56 presented the best correlation of CSM with neutralization (Extended Data Fig. 6a).

Furthermore, there is a strong and highly significant negative correlation between CXCR5 expression and the frequency of class switching on spike specific B cells. As CXCR5 facilitates homing to the germinal center where class switching primarily occurs ${ }^{34,47}$, this is highly suggestive of a causal association. The importance of class switching is further supported by the fact that the degree of class switching on spike specific $B$ cells at baseline has the strongest correlation with antibody titer, neutralization and ADCC at 28 days post vaccination (Fig. 6b). Importantly, individuals with a low fraction of CSM spike specific B cells displayed low neutralization and ADCC activity irrespective of prior infection. In addition, there is a strong positive correlation between CXCR3 expression and class switching at baseline, suggesting an association with B cell activation (Fig. 6a).

As increasing age is a known risk factor in both COVID-19 disease severity ${ }^{48}$ and reduced vaccine responsiveness ${ }^{2,17,49}$, we examined the impact of participant age on the spike specific $B$ cell response to vaccination. Notably, we observed significant negative associations between age and the activation of Spike+ memory B cells at day 28 and 56 (Fig. 6c), suggesting an age-related decline in B cell stimulation following vaccination. Finally, ten Spike+ memory B cell parameters were included as predictor variables in a least absolute shrinkage and selection operator (LASSO) regression model. The strongest predictor for the response variable of neutralization activity was prior infection, and an additional three $B$ cell parameters were chosen as relevant. All three have a positive effect and are listed in decreasing order (Fig. 6d): Spike+RBD+ memory B cell frequency, the degree of class switching (Spike+CSM), expression levels of CXCR3 (Spike+CXCR3). The LASSO coefficient paths plot and optimal penalty parameter selection plot are shown in the Extended Data Fig. 6b,c.

\section{Discussion}

This is the first study to characterize the memory B cell response to the Johnson and Johnson Ad26.COV2.S vaccine. We assess the capacity of a single-dose of the Ad26.COV2.S vaccine to induce specific B cell memory responses in a cohort that includes previously SARS-CoV-2 infected and naïve participants. Vaccination resulted in a 2-3-fold expansion of spike and RBD specific memory B cells that were durable for three months in both groups, like other vaccines investigated to date $2,25,39,50$. B cell memory in the naïve participants remained significantly reduced but eventually reached the baseline levels of the infected group. Notably, the magnitude of B cell response directly correlated with SARS-CoV2 spike specific antibody titer both prior to and following vaccination, validating the use of $B$ cell baits to monitor memory B cell responses.

These data build on recent work showing that the Ad26.COV2.S vaccine elicits functional antibody and T cell responses to SARS-CoV-2 and emerging variants ${ }^{14-16}$. These studies did not, however, examine the memory B cell response. Previous work on mRNA SARS-CoV-2 vaccines also found differences in the 
magnitude of specific memory B cells induced in naïve and previously infected individuals. Interestingly, for these vaccines, the levels observed in naïve individuals only reach the baseline level of the previously infected groups after the second dose ${ }^{2,25,50}$. By contrast, in this study, SARS-CoV-2 specific memory B cells reached this level in naïve individuals after a single dose of the Ad26.COV2.S vaccine. In addition, phenotyping of the SARS-CoV-2 specific memory B cell response revealed striking differences that correlated directly with antibody titer and functional activity and provided a unique mechanistic insight into how prior infection changes the vaccine response.

Most studies investigating the humoral response to vaccination have focused more on the antibody characteristics rather than on the B cells generating them. However, the B cell memory compartment is vital for antibody recall responses as it is maintained for long periods after infection and may be called upon to: respond to secondary challenge; boost antibody titers in response to high viral load; and adapt to a heterologous secondary challenge, such as with variants of concern, during which memory may be drawn back into germinal centers for further affinity maturation and class switching ${ }^{51}$. Therefore, understand the type of B cell immune memory elicited by vaccination with/without prior infection can help inform how individuals may respond to subsequent infection or vaccination. Memory B cell responses to SARS-CoV-2 infection can persist for at least five to eight months post-infection ${ }^{21-24}$. This is consistent with the observed positive correlation between the pre-vaccine frequency of SARS-CoV-2 specific memory B cells and antibody titers in the infected group. However, the fact that we observed a similar trend in the naïve group was somewhat unexpected. The antibody titer and spike specific B cell frequency were low in these individuals and only just above background levels. However, because the two independent measures correlate, they likely reveal pre-existing cross-reactive memory B cells in these individuals. This may be explained by the conserved nature of spike proteins amongst other common coronaviruses $^{5}$. In addition, SARS-CoV-2 binding naïve B cells have been observed in SARS-CoV-2-naïve individuals, suggesting that germline-encoded antibodies might have some cross-reactivity ${ }^{25,52}$. As new SARS-CoV-2 variants continue to emerge, the cross-reactivity of memory B cells is likely to be important in how prior infection and/or vaccination impacts immunity.

Despite this potential cross-reactivity, the B cells we observed in naïve individuals had a significantly greater IgM memory phenotype than the infected group, which is a characteristic of a primary response to an antigen ${ }^{35}$. Additionally, CXCR3+ expression was extremely low compared to the infected group, consistent with data showing this marker is elevated following persistent antigen exposure ${ }^{39}$. Thus, it seems likely that both cross-reactive and de novo $B$ cell responses emerged on vaccination. Going forward, phenotyping memory B cells in this way can help to tease apart the B cell response to subsequent SARS-CoV-2 variants and modified vaccines.

Although vaccination increased CXCR3 expression in naïve individuals, it returned to baseline levels by day 56. In previously infected individuals, however, a consistent fraction of spike specific B cells expressed CXCR3 at all timepoints. This receptor facilitates homing to inflamed respiratory mucosae and its expression is IFN- $\gamma$-dependent involving the IFN- - -induced transcription factor T-bet ${ }^{39,42,43}$. This 
suggests that memory B cells induced by prior infection may be better primed to home to the lung than those generated by single dose vaccination. Importantly, there was a strong correlation between the AlM+ CD8+ $T$ cell and Spike+CXCR3+ memory B cell responses. Antigen-specific CD8+ T cells can either produce IFN- $\gamma$ themselves or induce its production in CD4+ Tfh cells which then drives CXCR3 expression and class switching in germinal center $B$ cells ${ }^{43,53}$. Vaccine boosting does increase B cell CXCR3 expression ${ }^{39}$ and although this has not been measured directly for COVID-19 vaccines, Pape et al. (2021) describe increased atypical memory B cells following mRNA vaccine boosting. Atypical B cells express Tbet and $\mathrm{CXCR} 3^{39,54,55}$. Together these data suggest a mechanism through which booster vaccination could improve SARS-CoV-2 specific memory B cell homing to lung mucosae.

Another striking phenotypic difference in the $B$ cell response to vaccination between the naïve and infected group concerns CXCR5 expression, which controls germinal center homing ${ }^{40,41}$. Importantly, expression levels of this marker were similar between groups at baseline, indicating that the rapid downregulation of CXCR5 on SARS-CoV-2 specific memory B cells in previously infected individuals was explicitly in response to vaccination. $B$ cells reduce their CXCR5 expression upon encountering their cognate antigen ${ }^{56}$. We hypothesize that this phenomenon either reflects a spill-over of spike specific $B$ cells from secondary lymphoid organs s $^{25,32}$ or the enhanced trafficking of CXCR5+B cells to germinal centers. Importantly, both are suggestive of increased germinal center activity following vaccination. This hypothesis is strongly supported by the inverse correlation after vaccination between CXCR5 expression and class-switching on SARS-CoV-2 specific memory B cells and both antibody neutralization and ADCC activity. In addition, the AIM + CD4+ and cTfh also correlated with memory B cell class switching, a critical interaction which has also been demonstrated in natural infection ${ }^{44}$. Vaccine efficacy is primarily based on neutralization activity ${ }^{10,11,57}$, and these data provide mechanistic insight into how a differential memory $B$ cell response to vaccination leads to higher neutralization titers in individuals with prior exposure. It will be essential to determine how this response compares to booster doses given to naïve individuals.

Using a LASSO approach, we identified class switching as the strongest memory B cell predictor of the antibody response to vaccination. The importance of class switching is supported by the link between rapid class-switched antibody responses and a positive COVID-19 disease outcome in unvaccinated individuals ${ }^{12}$. Class-switched memory $B$ cells are more adept at mounting a rapid antibody response upon subsequent infection or antigen exposure $25,58,59$. In line with this, we observed significant correlations between the degree of class-switching on SARS-CoV-2 memory B cells at baseline and the quantity and quality of the antibody response measured at days 28 and 56 . This is consistent with increased class switching in previously infected participants observed with mRNA vaccines, especially following the first dose ${ }^{2,25,50}$. Therefore, prior exposure affords a significant advantage to the extent of class-switched memory B cells induced by single-dose vaccination. Again, it will be essential to test if boosting naïve individuals can achieve the same degree of class switching as natural infection. 
Another informative phenotypic marker from this study was CD21, which was included to measure B cell activation following vaccination ${ }^{36-39}$. The clear spike in SARS-CoV-2 specific B cell activation on day 28 indicated that vaccination achieved similar stimulation in naïve and infected individuals. This is consistent with human influenza and yellow fever vaccination experiments that found a peak of CD21 $1^{\mathrm{lo}}$ antigen-specific $B$ cells at day $14-28$ post vaccination ${ }^{60,61}$. These studies also found evidence that CD $21^{10}$ $B$ cells were recent germinal center immigrants and preferentially developed into long-lived plasma cells. Therefore, the negative correlation between age and the frequency of $C D 21^{10} \mathrm{~B}$ cells, irrespective of prior exposure, provides potential mechanistic explanations for reduced vaccine efficiency observed in older individuals. Sampling additional early timepoints following vaccination may provide further insights into the initial response kinetics of B cell activation and uncover the impact of known co-morbidities such as untreated HIV or diabetes ${ }^{62}$. In addition, investigating the presence of correlations between CD21 expression and $B$ cell regulatory markers may provide novel opportunities to modulate B cell activation during vaccination.

A limitation of this study is that we used spike and RBD bait proteins based on the ancestral D614G virus only. Therefore, we may have missed memory B cell responses present in individuals infected with the Beta variant. However, since the vaccine is based on the ancestral virus, we believe this reagent was suitable for studying the vaccine response. Pape et al. (2021) found that $62-74 \%$ of the memory B cells specific to ancestral spike protein were cross-reactive to Beta variant spike following mRNA vaccination. After vaccination, the antibody response in the naïve group was reactive to both the ancestral D614G and Beta variant spike proteins, facilitating similar binding and $A D C C$, but this did not translate to equally reactive neutralization capacity.

By combining antibody and $T$ cell measures with expanded memory B cell phenotyping that included activation and homing markers, we gain mechanistic insight into the post-vaccination memory $B$ cell response. This response is significantly more robust in participants with a history of COVID-19 and was maintained for the study duration (84 days). Of note we point out an overall reduction in memory $B$ cell activation associated with age, regardless of infection history. It would be important to compare the longterm memory duration in these groups and to study the effectiveness of boosting regimens, both regarding the memory response but also efficacy against new variants and breakthrough infections.

\section{Materials And Methods}

\section{Participants and longitudinal follow up}

Our study was based on the SISONKE Phase $3 \mathrm{~b}$ trial, in which health care workers were given single dose Johnson and Johnson Ad26.COV2.S between 17 February and 26 March 2021. Participants were recruited at Groote Schuur Hospital (Cape Town, Western Cape, South Africa), to form part of a longitudinal cohort of 400 health care workers. We included 30 participants from this cohort in this study and these included 10 naïve and 20 previously infected vaccinees. Of the previously infected, nine were infected during the first wave (May to August 2020 with the ancestral D614G virus); five during the 
second wave (November 2020 to January 2021 with the Beta variant) and six were infected but the infection timing could not be confirmed. Infection history was confirmed by laboratory PCR and/or serology testing. The participant demographic and clinical data were summarized in Extended Data Table 1. The study was approved by the University of Cape Town Human Research Ethics Committee (HREC 190/2020 and 209/2020), the University of the Witwatersrand Human Research Ethics Committee (Medical; no M210429). Written informed consent was obtained from all participants.

\section{Serology screening}

Serum samples were screened for antibodies specific for the SARS-CoV-2 spike and nucleocapsid $(\mathrm{N})$ proteins. This was done using the Roche Elecsys ${ }^{\circledR} S$ and $N$ test (Roche Diagnostics, $\mathrm{GmbH}$ ). Samples were analyzed on a Cobas e801 instrument and a result $\geq 0.8 \mathrm{U} / \mathrm{mL}$ was considered positive in the $S$ assay, and $\geq 1.0 \mathrm{U} / \mathrm{mL}$ positive in the $\mathrm{N}$ assay, according to the manufacturer's instructions.

\section{Peripheral blood mononuclear cell (PBMC) isolation}

All bloods were collected into heparin tubes and processed within four hours of collection. A Ficoll-Paque (Amersham Biosciences, Little Chalfont, UK) density gradient sedimentation was used to separate the PBMCs as per manufacturer's instructions. PBMCs were cryopreserved in freezing media containing $10 \%$ DMSO in heat inactivated fetal bovine serum (FBS, ThermoFischer Scientific) and stored in liquid nitrogen.

\section{SARS-CoV-2 spike and receptor binding domain (RBD) proteins}

The SARS-CoV-2 D614G and Beta variant spike and receptor binding domain (RBD) proteins were recombinantly expressed in Human Embryonic Kidney (HEK) 293F suspension cells. Following a six-day culture at $37^{\circ} \mathrm{C}, 70 \%$ humidity and $10 \% \mathrm{CO}_{2}$, the expressed viral proteins were purified on a nickel resin, followed by size exclusion chromatography, and stored at $-80^{\circ} \mathrm{C}$ until use.

\section{SARS-CoV-2 spike and RBD B cell bait staining and flow cytometry}

Spike and RBD biotinylated bait proteins were labeled with Streptavidin-APC (SA-APC) or Streptavidin-PE (SA-PE) at a molar ratio of 4 (bait) to 1 (SA-APC or SA-PE) as described ${ }^{2}$. Frozen PBMCs were thawed and rested for 1 hour in a $37^{\circ} \mathrm{C}, 5 \% \mathrm{CO}_{2}$ incubator. After resting a minimum of $5 \times 10^{6}$ to $1 \times 10^{7}$ cells were stained with a surface stain antibody mix (Extended Data Table 2, markers 1 to 12) for 20 minutes at room temperature, washed twice with $1 \mathrm{ml}$ PBS and then stained with the SA-APC and SA-PE labeled bait proteins for an additional 30 minutes at room temperature. All antibody and bait staining mixes were prepared in 10\% FCS in PBS using the dilution factors indicated in Extended Data Table 2, with a final volume of $25 \mu \mathrm{l}$ per set of cells to be stained. The cells were washed twice with $1 \mathrm{ml}$ PBS and finally suspended in $0.5 \mathrm{ml}$ PBS, kept on ice, and acquired on a BD FACS Aria Fusion III and data analyzed using FlowJo version 9.9.6 (Tree Star).

\section{Activation induced marker (AIM) assay}


Cryopreserved PBMCs were thawed, washed, and rested in RPMI 1640 containing 10\% heat-inactivated FCS for 4 hours prior to stimulation. PBMCs were seeded in a 96-well V-bottom plate at $\sim 1 \times 10^{6}$ PBMCs per well and stimulated with a SARS-CoV-2 spike peptide pool (15mers overlapping by 10 amino acids) based on the full ancestral protein (Miltenyi, $1 \mu \mathrm{g} / \mathrm{mL}$ in distilled water). As a negative control, PBMCs were stimulated with media alone. After 16 hours of stimulation, cells were washed, stained with LIVE/DEAD ${ }^{\text {TM }}$ Fixable Near Infrared Stain (Invitrogen, Carlsbad, CA, USA) and subsequently stained with the surface marker antibodies (Extended Data Table 3). Finally, cells were washed and fixed in CellFix (BD Biosciences). Samples were acquired on a BD LSRII flow cytometer and analyzed using FlowJo (v10, FlowJo, Ashland, OR, USA). Cells were gated on singlets, lymphocytes, live CD3+ cells, and CD4+ or CD8+ T cells. AIM+ cells were those co-expressing OX40 and CD137 (for CD4+) or CD69 and CD137 (for CD8+), while the AIM+ cTfh population was defined as CXCR5+CD45RA- CD4+ T cells co-expressing OX40 and CD25. Results are expressed as the frequency of AIM+ CD4+ or CD8+ T cells, and AIM+cTfh expressed as a frequency of total CD4+ T cells. AIM+ responses presented are background subtracted (from the frequency of AIM+ cells in the unstimulated sample), and the threshold for AIM positivity was defined as $>1.5 x$ the unstimulated sample and $>0.01 \%$.

\section{Spike specific Enzyme linked immunosorbent assay (ELISA)}

96-well ELISA plates were coated overnight at $4^{\circ} \mathrm{C}$ with viral spike protein at $2 \mu \mathrm{g} / \mathrm{ml}$. Plates were blocked with $5 \%$ skimmed milk powder in PBS with $0.05 \%$ Tween 20 . All plasma was diluted 1:100 in blocking buffer and added to the plate, after which the plates were incubated with secondary antibody at 1:3000 in blocking buffer. The signal was developed with TMB substrate (ThermoFischer Scientific) and $1 \mathrm{M} \mathrm{H}_{2} \mathrm{SO}_{4}$ was used to stop the reaction. Absorbance was measured at $450 \mathrm{~nm}$. All assays included monoclonal antibodies CR3022 and BD23 as positive controls and palivizumab as a negative control.

\section{Pseudovirus neutralization assay}

Two SARS-CoV-2 pseudotyped lentiviruses were prepared by co-transfecting a HEK293T cell line with either the ancestral virus spike (D614G) or the Beta spike (L18F, D80A, D215G, K417N, E484K, N501Y, D614G, A701V, 242-244 del) plasmids and a firefly luciferase lentivirus backbone plasmid. Participant plasmas were heat inactivated and incubated with each of the pseudotyped SARS-CoV-2 viruses for 1

hour at $37^{\circ} \mathrm{C}, 5 \% \mathrm{CO}_{2}$. Following this incubation, the pseudoviruses were incubated with $1 \times 10^{4} \mathrm{HEK} 293 \mathrm{~T}$ cells overexpressing the ACE-2 surface receptor for 72 hours at $37^{\circ} \mathrm{C}, 5 \% \mathrm{CO}_{2}$. Any cells that were successfully infected by the pseudoviruses, were detected by luminometry of the luciferase gene. The CB6 monoclonal antibody was used as a positive control for neutralization.

\section{Antibody dependent cellular cytotoxicity (ADCC)}

An indirect measure of ADCC was performed by assessing the ability of participant plasma to crosslink the FcyRIIla (CD16) surface receptor of Jurkat-Lucia ${ }^{\text {TM }}$ NFAT-CD16 cells and SARS-CoV-2 spike expressing HEK293T cells. The HEK293T cells were transfected with $5 \mu \mathrm{g}$ of SARS-CoV-2 ancestral virus 
(D614G) or Beta variant (L18F, D80A, D215G, K417N, E484K, N501Y, D614G, A701V, 242-244 del) spike plasmids using PEI-MAX 40000 (Polysciences) and incubated for 2 days at $37^{\circ} \mathrm{C}, 5 \% \mathrm{CO}_{2}$. Spike expression was confirmed by flow cytometry, using anti-spike CR3022 or P2B-2F6 and anti-lgG-APC staining. Heat inactivated plasma were diluted 1:100 in R10 media (RPMI 1640 media with 10\% FCS and 1\% Penicillin/Streptomycin (Gibco, Gaithersburg, MD)). Control antibodies were used at $100 \mu \mathrm{g} / \mathrm{ml}$ and $1 \times 10^{5}$ spike transfected HEK293T cells/well, of a 96 well culture plate and were incubated with the antibody treated media for 1 hour at $37^{\circ} \mathrm{C}, 5 \% \mathrm{CO}_{2}$. The Jurkat-Lucia ${ }^{\mathrm{TM}}$ NFAT-CD16 cells (Invivogen) were added at $2 \times 10^{5}$ cells/well and incubated for a further 24 hours. A $20 \mu$ aliquot of the culture supernatant was transferred to a white 96 well plate containing $50 \mu \mathrm{l} /$ well QUANTI-Luc secreted luciferase and read immediately using a Victor 3 luminometer set to a $1 \mathrm{sec}$ integration time. A no antibody control was used to subtract background luminescence. The monoclonal antibodies: Palivizumab served as negative control, CR3022 as positive control and P2B-2F6 to differentiate the Beta from ancestral (D614G) variant. A $1 x$ cell stimulation cocktail (ThermoFischer Scientific, Oslo, Norway) and $2 \mu \mathrm{g} / \mathrm{ml}$ ionomycin in R10 media was added and served as a positive control by inducing the transgene.

\section{Statistical analysis}

All analyses were performed in Prism (v9; GraphPad Software Inc., San Diego, CA, USA), except for the LASSO analysis, which was performed in the statistical software $\mathrm{R}^{63}$. Nonparametric tests were used throughout, with Mann-Whitney and Wilcoxon tests used for unmatched and paired samples, respectively. All correlations reported are nonparametric Spearman rank correlations. $P$ values less than 0.05 were considered statistically significant and denoted by $* \leq 0.05 ; * \star<0.01 ; * \star *<0.001$ and $* \star \star *<0.0001$. The influence of several potential Spike+ memory B cell predictor variables () on the SARS-CoV-2 neutralization was assessed by the so-called least absolute shrinkage and selection operator (LASSO) principle ${ }^{64}$, based on scaled covariates, and fitting a log-linear model via the glmnet R package ${ }^{65,66}$. For this method, the optimal tuning parameter, which reflects the amount of penalization and, hence, controls variable selection, is determined via 10-fold cross-validation based on the model's deviance (see

\section{Extended Data Fig. 6b,c).}

\section{Declarations}

\section{Acknowledgements}

The authors thank the study participants and their families, and the clinical staff and personnel at Groote Schuur Hospital in Cape Town for their support and dedication. We thank Amkele Ngomti, Richard Baguma and Elloise du Toit for assistance with sample processing, storage, and shipment. We would also like to thank Drs Elise Landais and Helena Almaguer from Scripps Research for sharing viral spike and RBD reagents. We thank the informal 5 501Y.V2 consortium of South African scientists, chaired by Drs Willem Hanekom and Tulio de Oliveira for suggestions.

\section{Funding}


Research reported in this publication was supported by the South African Medical Research Council with funds received from the Department of Science and Innovation. The content and findings reported are the sole deduction, view and responsibility of the researchers and do not reflect the official position and sentiments of the SAMRC. W.A.B. is supported by the EDCTP2 program of the European Union's Horizon 2020 program (TMA2016SF-1535-CaTCH-22), the Wellcome Centre for Infectious Diseases Research in Africa (CIDRI-Africa) which is supported by core funding from the Wellcome Trust (203135/Z/16/Z) and the Poliomyelitis Research Foundation (PRF 21/65). P.L.M. is supported by the South African Research Chairs Initiative of the Department of Science and Innovation and National Research Foundation of South Africa, the SA Medical Research Council SHIP program, and the Centre for the AIDS Program of Research (CAPRISA). S.I.R. is a L'Oreal/UNESCO Women in Science South Africa Young Talents awardee. Related research by the authors is conducted as part of the DST-NRF Centre of Excellence in HIV Prevention, which is supported by the Department of Science and Technology and the National Research Foundation.

\section{Contributions:}

N.A.B.N., W.A.B, P.L.M. and A.L. designed the study. R.G.E.K. generated B cell data and wrote the manuscript. W.A.B., C.R., R.K., A.L., analyzed the data and wrote the manuscript. R.K. and N.B. generated T cell data. S.I.R., T.M.G., T.H., N.P.M., Z.M. and N.M. performed ELISAs, neutralization assays and ADCC assays. M.M. and S.S. managed the HCW cohort and contributed clinical samples and data. A.Goga, N.G., L.G.B. and G.G. established and led the Sisonke vaccine study. N.A.B.N. established and led the HCW cohort and contributed samples. A.Groll assisted with statistical analyses. All authors reviewed and edited the manuscript.

\section{Competing interests:}

The authors declare no competing interests.

\section{References}

1. Bos, R., et al. Ad26 vector-based COVID-19 vaccine encoding a prefusion-stabilized SARS-CoV-2 Spike immunogen induces potent humoral and cellular immune responses. npj Vaccines 5(2020).

2. Goel, R.R., et al. Distinct antibody and memory B cell responses in SARS-CoV-2 naive and recovered individuals following mRNA vaccination. Sci Immunol 6(2021).

3. Sadoff, J., et al. Interim Results of a Phase 1-2a Trial of Ad26.COV2.S Covid-19 Vaccine. N Engl J Med 384, 1824-1835 (2021).

4. Ziegler, C.G.K., et al. SARS-CoV-2 Receptor ACE2 Is an Interferon-Stimulated Gene in Human Airway Epithelial Cells and Is Detected in Specific Cell Subsets across Tissues. Cell 181, 1016-1035 e1019 (2020).

5. Ahmed, S.F., Quadeer, A.A. \& McKay, M.R. Preliminary Identification of Potential Vaccine Targets for the COVID-19 Coronavirus (SARS-CoV-2) Based on SARS-CoV Immunological Studies. Viruses 
12(2020).

6. Barnes, C.O., et al. SARS-CoV-2 neutralizing antibody structures inform therapeutic strategies. Nature 588, 682-687 (2020).

7. Brouwer, P.J.M., et al. Potent neutralizing antibodies from COVID-19 patients define multiple targets of vulnerability. Science $369,643-650$ (2020).

8. McCallum, M., et al. N-terminal domain antigenic mapping reveals a site of vulnerability for SARSCoV-2. Cell 184, 2332-2347 e2316 (2021).

9. Piccoli, L., et al. Mapping Neutralizing and Immunodominant Sites on the SARS-CoV-2 Spike Receptor-Binding Domain by Structure-Guided High-Resolution Serology. Cell 183, 1024-1042 e1021 (2020).

10. Feng, S., et al. Correlates of protection against symptomatic and asymptomatic SARS-CoV-2 infection. Nat Med 27, 2032-2040 (2021).

11. Hall, V.J., et al. SARS-CoV-2 infection rates of antibody-positive compared with antibody-negative health-care workers in England: a large, multicentre, prospective cohort study (SIREN). Lancet 397, 1459-1469 (2021).

12. Zohar, T., et al. Compromised Humoral Functional Evolution Tracks with SARS-CoV-2 Mortality. Cell 183, 1508-1519 e1512 (2020).

13. Mendonca, S.A., Lorincz, R., Boucher, P. \& Curiel, D.T. Adenoviral vector vaccine platforms in the SARS-CoV-2 pandemic. NPJ Vaccines 6, 97 (2021).

14. Alter, G., et al. Immunogenicity of Ad26.COV2.S vaccine against SARS-CoV-2 variants in humans. Nature 596, 268-272 (2021).

15. Barouch, D.H., et al. Durable Humoral and Cellular Immune Responses 8 Months after Ad26.COV2.S Vaccination. N Engl J Med 385, 951-953 (2021).

16. Keeton, R., et al. Prior infection with SARS-CoV-2 boosts and broadens Ad26.COV2.S immunogenicity in a variant-dependent manner. Cell Host Microbe 29, 1611-1619 e1615 (2021).

17. Pegu, A., et al. Durability of mRNA-1273 vaccine-induced antibodies against SARS-CoV-2 variants. Science 373, 1372-1377 (2021).

18. Tada, T., et al. Comparison of Neutralizing Antibody Titers Elicited by mRNA and Adenoviral Vector Vaccine against SARS-CoV-2 Variants. bioRxiv (2021).

19. Akkaya, M., Kwak, K. \& Pierce, S.K. B cell memory: building two walls of protection against pathogens. Nat Rev Immunol 20, 229-238 (2020).

20. Kurosaki, T., Kometani, K. \& Ise, W. Memory B cells. Nat Rev Immunol 15, 149-159 (2015).

21. Dan, J.M., et al. Immunological memory to SARS-CoV-2 assessed for up to 8 months after infection. Science 371(2021).

22. Gaebler, C., et al. Evolution of antibody immunity to SARS-CoV-2. Nature 591, 639-644 (2021).

23. Hartley, G.E., et al. Rapid generation of durable B cell memory to SARS-CoV-2 spike and nucleocapsid proteins in COVID-19 and convalescence. Sci Immuno/ 5(2020). 
24. Turner, J.S., et al. SARS-CoV-2 infection induces long-lived bone marrow plasma cells in humans. Nature 595, 421-425 (2021).

25. Pape, K.A., et al. High-affinity memory B cells induced by SARS-CoV-2 infection produce more plasmablasts and atypical memory B cells than those primed by mRNA vaccines. Cell Rep 37, 109823 (2021).

26. Manisty, C., et al. Antibody response to first BNT162b2 dose in previously SARS-CoV-2-infected individuals. Lancet 397, 1057-1058 (2021).

27. Saadat, S., et al. Binding and Neutralization Antibody Titers After a Single Vaccine Dose in Health Care Workers Previously Infected With SARS-CoV-2. JAMA 325, 1467-1469 (2021).

28. Stamatatos, L., et al. mRNA vaccination boosts cross-variant neutralizing antibodies elicited by SARS-CoV-2 infection. Science (2021).

29. Vanshylla, K., et al. Kinetics and correlates of the neutralizing antibody response to SARS-CoV-2 infection in humans. Cell Host Microbe 29, 917-929 e914 (2021).

30. Wang, Z., et al. mRNA vaccine-elicited antibodies to SARS-CoV-2 and circulating variants. Nature 592, 616-622 (2021).

31. Takuva, S., et al. Thromboembolic Events in the South African Ad26.COV2.S Vaccine Study. N Engl J Med 385, 570-571 (2021).

32. Blink, E.J., et al. Early appearance of germinal center-derived memory B cells and plasma cells in blood after primary immunization. J Exp Med 201, 545-554 (2005).

33. Schroeder, H.W., Jr. \& Cavacini, L. Structure and function of immunoglobulins. J Allergy Clin Immunol 125, S41-52 (2010).

34. Stavnezer, J., Guikema, J.E. \& Schrader, C.E. Mechanism and regulation of class switch recombination. Annu Rev Immuno/ 26, 261-292 (2008).

35. St John, A.L. \& Rathore, A.P.S. Adaptive immune responses to primary and secondary dengue virus infections. Nat Rev Immunol 19, 218-230 (2019).

36. Ellebedy, A.H., et al. Defining antigen-specific plasmablast and memory B cell subsets in human blood after viral infection or vaccination. Nat Immunol 17, 1226-1234 (2016).

37. Louis, K., et al. T-bet+CD27+CD21- B cells poised for plasma cell differentiation during antibodymediated rejection of kidney transplants. JCl Insight 6(2021).

38. Ogega, C.O., et al. Durable SARS-CoV-2 B cell immunity after mild or severe disease. J Clin Invest 131(2021).

39. Sutton, H.J., et al. Atypical B cells are part of an alternative lineage of B cells that participates in responses to vaccination and infection in humans. Cell Rep 34, 108684 (2021).

40. Lam, J.H., Smith, F.L. \& Baumgarth, N. B Cell Activation and Response Regulation During Viral Infections. Viral Immunol 33, 294-306 (2020).

41. Okada, T., et al. Chemokine requirements for B cell entry to lymph nodes and Peyer's patches. $J$ Exp Med 196, 65-75 (2002). 
42. Onodera, T., et al. Memory B cells in the lung participate in protective humoral immune responses to pulmonary influenza virus reinfection. Proc Natl Acad Sci U S A 109, 2485-2490 (2012).

43. Serre, K., et al. CD8 T cells induce T-bet-dependent migration toward CXCR3 ligands by differentiated B cells produced during responses to alum-protein vaccines. Blood 120, 4552-4559 (2012).

44. Pusnik, J., et al. Memory B cells targeting SARS-CoV-2 spike protein and their dependence on CD4(+) T cell help. Cell Rep 35, 109320 (2021).

45. Jung, J.H., et al. SARS-CoV-2-specific T cell memory is sustained in COVID-19 convalescent patients for 10 months with successful development of stem cell-like memory T cells. Nat Commun 12, 4043 (2021).

46. Reiss, S., et al. Comparative analysis of activation induced marker (AIM) assays for sensitive identification of antigen-specific CD4 T cells. PLoS One 12, e0186998 (2017).

47. Gitlin, A.D., et al. Independent Roles of Switching and Hypermutation in the Development and Persistence of B Lymphocyte Memory. Immunity 44, 769-781 (2016).

48. Ho, F.K., et al. Is older age associated with COVID-19 mortality in the absence of other risk factors? General population cohort study of 470,034 participants. PLoS One 15, e0241824 (2020).

49. Ciabattini, A., et al. Vaccination in the elderly: The challenge of immune changes with aging. Semin Immunol 40, 83-94 (2018).

50. Mazzoni, A., et al. First-dose mRNA vaccination is sufficient to reactivate immunological memory to SARS-CoV-2 in subjects who have recovered from COVID-19. J Clin Invest 131(2021).

51. McHeyzer-Williams, M., Okitsu, S., Wang, N. \& McHeyzer-Williams, L. Molecular programming of B cell memory. Nat Rev Immunol 12, 24-34 (2011).

52. Sette, A. \& Crotty, S. Adaptive immunity to SARS-CoV-2 and COVID-19. Cell 184, 861-880 (2021).

53. Mohr, E., et al. IFN-\{gamma\} produced by CD8 T cells induces T-bet-dependent and -independent class switching in B cells in responses to alum-precipitated protein vaccine. Proc Natl Acad Sci USA 107, 17292-17297 (2010).

54. Jenks, S.A., et al. Distinct Effector B Cells Induced by Unregulated Toll-like Receptor 7 Contribute to Pathogenic Responses in Systemic Lupus Erythematosus. Immunity 49, 725-739 e726 (2018).

55. Woodruff, M.C., et al. Extrafollicular B cell responses correlate with neutralizing antibodies and morbidity in COVID-19. Nat Immunol 21, 1506-1516 (2020).

56. Lam, J.H. \& Baumgarth, N. The Multifaceted B Cell Response to Influenza Virus. J Immuno/ 202, 351-359 (2019).

57. Khoury, J., et al. COVID-19 vaccine - Long term immune decline and breakthrough infections. Vaccine 39, 6984-6989 (2021).

58. Pape, K.A., Taylor, J.J., Maul, R.W., Gearhart, P.J. \& Jenkins, M.K. Different B cell populations mediate early and late memory during an endogenous immune response. Science 331, 1203-1207 (2011).

59. Wrammert, J., et al. Rapid cloning of high-affinity human monoclonal antibodies against influenza virus. Nature 453, 667-671 (2008). 
60. Lau, D., et al. Low CD21 expression defines a population of recent germinal center graduates primed for plasma cell differentiation. Sci Immuno/ 2(2017).

61. Wec, A.Z., et al. Longitudinal dynamics of the human B cell response to the yellow fever 17D vaccine. Proc Natl Acad Sci U S A 117, 6675-6685 (2020).

62. Bhaskaran, K., et al. HIV infection and COVID-19 death: a population-based cohort analysis of UK primary care data and linked national death registrations within the OpenSAFELY platform. Lancet HIV 8, e24-e32 (2021).

63. Team, R.C. R: A language and environment for statistical computing. $R$ Stat Comp (2020).

64. Tibshirani, R. Regression Shrinkage and Selection Via the Lasso. J R Statistic Soc B 58, 267-288 (1996).

65. Friedman, J., Hastie, T. \& Tibshirani, R. Regularization Paths for Generalized Linear Models via Coordinate Descent. J Stat Softw 33, 1-22 (2010).

66. Muenchhoff, M., et al. Nonprogressing HIV-infected children share fundamental immunological features of nonpathogenic SIV infection. Sci Trans/ Med 8, 358ra125 (2016).

\section{Figures}


a
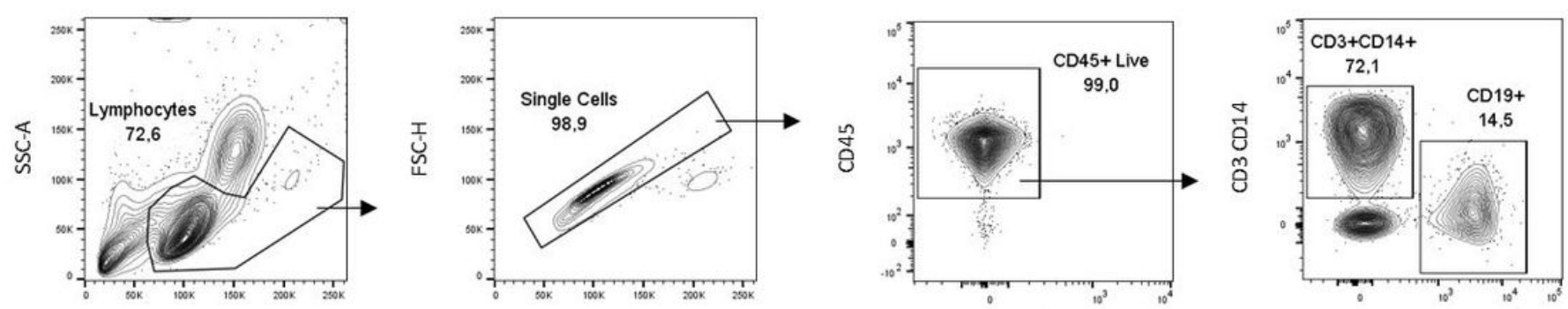

FSC-A

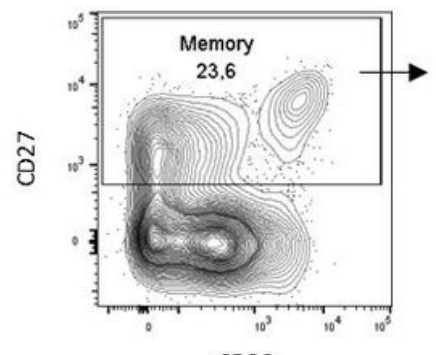

FSC-A

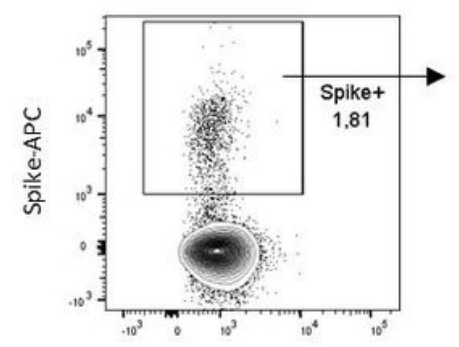

L/D

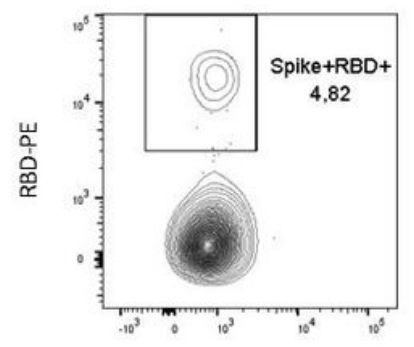

CD19

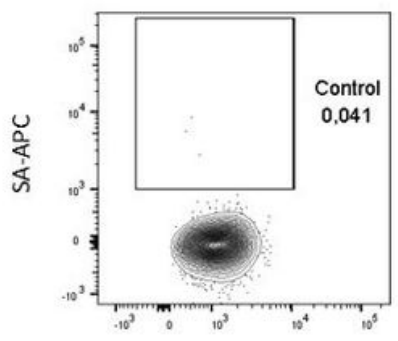

CD45

CD45

b

(x2.1)

(x2)

$(x 2.1) \quad(x 2.1)$

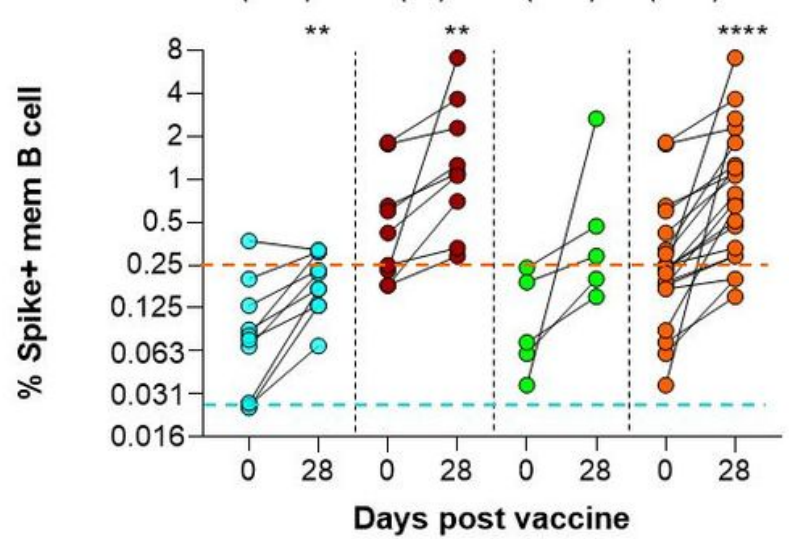

(x2.4)

$(x 2.6) \quad(x 4.5)$

(x3)

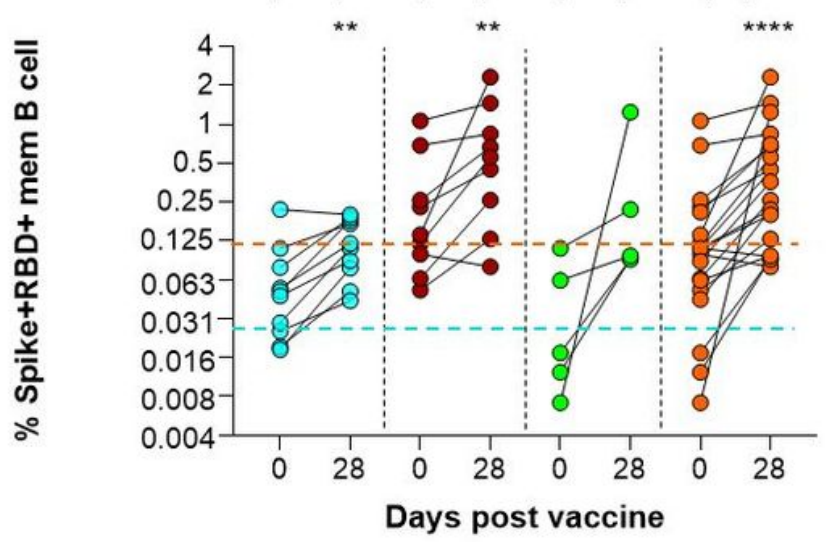

○ Naïve (10) $\bullet 1^{\text {st }}$ wave infection (9) $\bigcirc 2^{\text {nd }}$ wave infection (5) $\bullet \quad$ Infected - total (20)

\section{Figure 1}

Spike and receptor binding domain (RBD) specific memory B cell responses prior to (baseline) and 28 days post-vaccination. a, Gating strategy identifying the CD19+ B cells (top row) and subsequent memory (CD27+), Spike+ (Spike-APC) and Spike+RBD+ (RBD-PE) memory B cells (bottom row). The spike and RBD baits were both from the D614G ancestral virus. The final panel serves as a background control for Streptavidin-APC (SA-APC). All population frequencies are relative to the gated parent population in the previous plot. A dump channel removed CD3 and CD14 positive cells. b, The total Spike+ and Spike+RBD+ B cell memory responses were compared prior to (baseline) and at day 28 after vaccination. The fold increase of the response per group is indicated in brackets above each plot. The key indicates the COVID-19 infection history of vaccinated participants with sample number in brackets. The dashed lines represent the initial median response for the naïve (blue) and previously infected (orange) groups. Statistical analyses were performed using the Wilcoxon test for baseline and day 28 time points and Mann-Whitney for day 0 comparisons between groups. $P$ values are denoted by $* \leq 0.05$; $\star \star<0.01 ; * \star \star<$ 0.001 and $* * * *<0.0001$. 
a

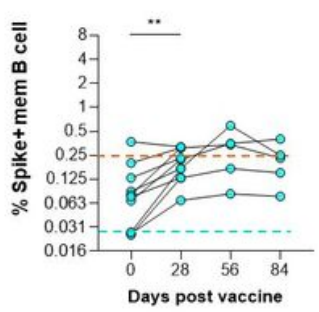

c

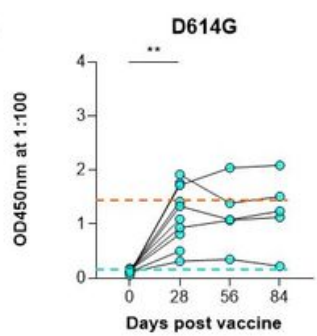

e

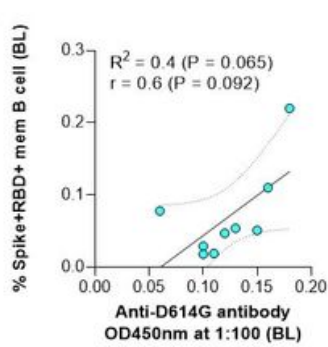

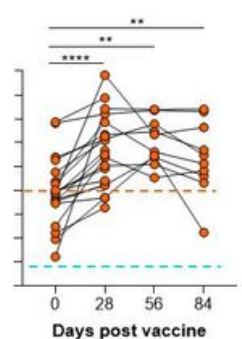
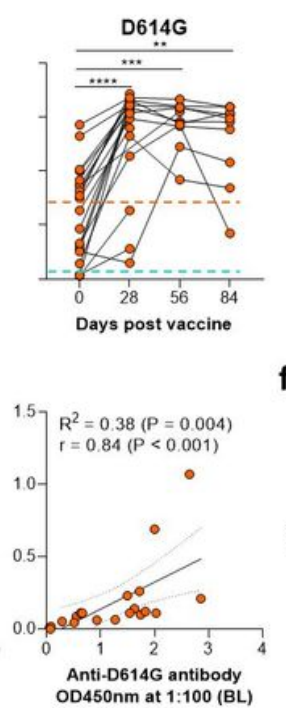

b
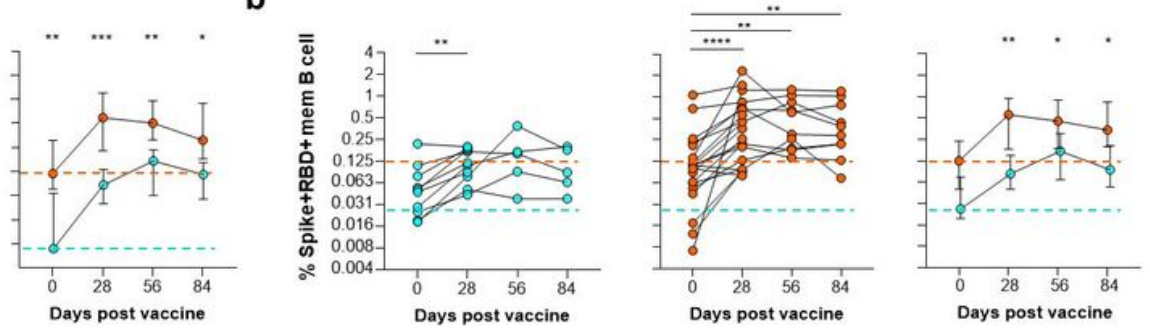

D614G

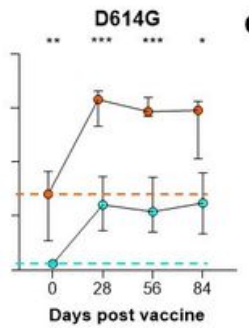

Beta
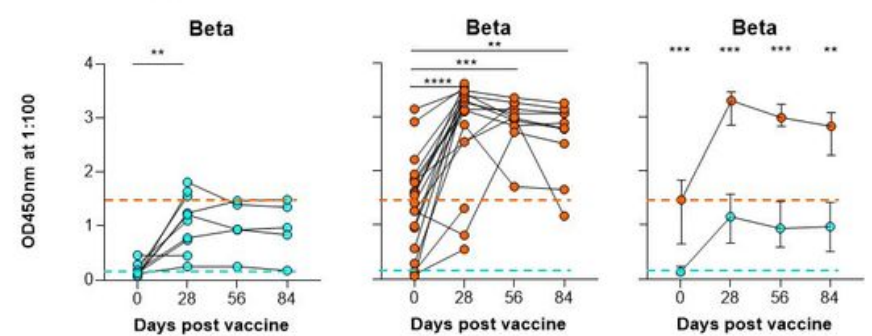

f
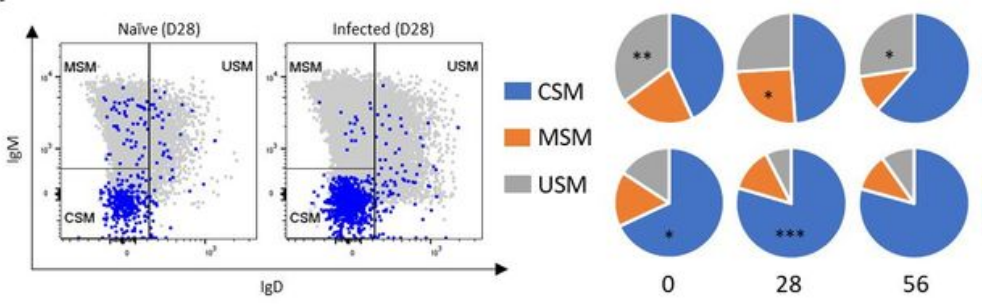

28 past vaccin

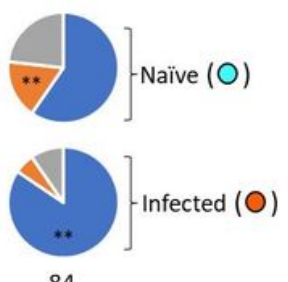

84

Figure 2

Longitudinal B cell memory and corresponding antibody responses to Spike and/or RBD. a, The Spike+ and $\mathbf{b}$, Spike+RBD+ memory B cell responses were tracked over a 3-month longitudinal follow up at 28day intervals. Individual longitudinal plots are followed by a median $\left({ }^{+} / .95 \% \mathrm{Cl}\right)$ longitudinal group plot, with the naïve response in blue and previously infected response in orange throughout. The dashed lines represent the initial median response for the naïve (blue) and previously infected (orange) groups. The corresponding longitudinal antibody responses specific for D614G spike in c, and Beta variant spike in d, are shown in a similar layout. e, Spearman non-parametric correlation of the Spike+RBD+ B cell memory versus D614G spike-specific antibody response at baseline (BL). f, Comparison of the extent of Spike+ class switched B cell memory between the naïve and previously infected groups. A representative Spike+ memory B cell population (blue) was overlaid onto the total B cell plot (grey) of lgM versus lgD for a naïve and infected vaccinee at day 28 . The resulting $\lg M-\lg D$ - class switched memory (CSM), $\lg M+\lg D-\lg M$ switched memory (MSM) and unswitched memory (USM) responses were compared as pie charts. Statistical analyses were performed using the Wilcoxon test for longitudinal time points versus day 0 and Mann-Whitney test between naïve and previously infected groups. $P$ values are denoted by $* \leq 0.05$; $*$ < $0.01 ; * \star \star<0.001$ and $* \star \star \star<0.0001$. 
a

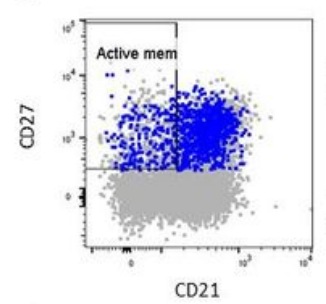

d

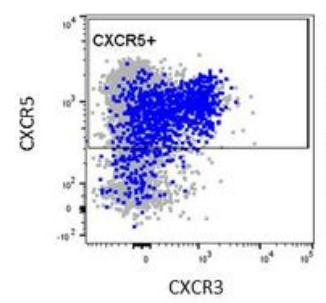

g

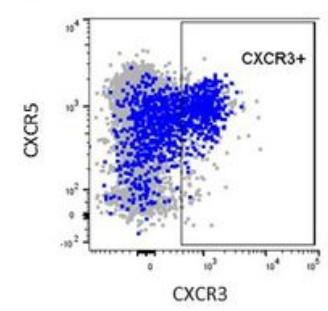

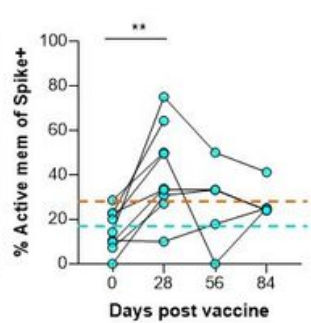
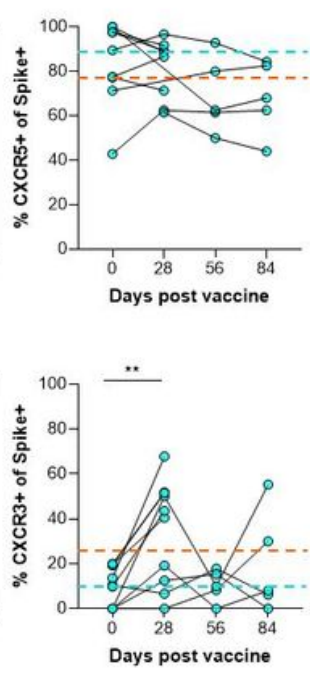

Naïve (O)
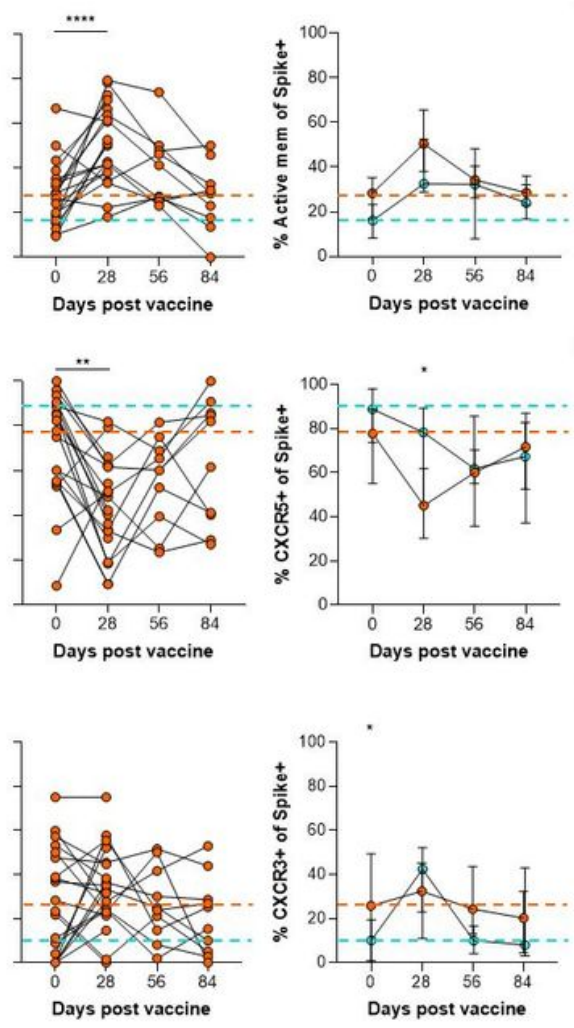

h
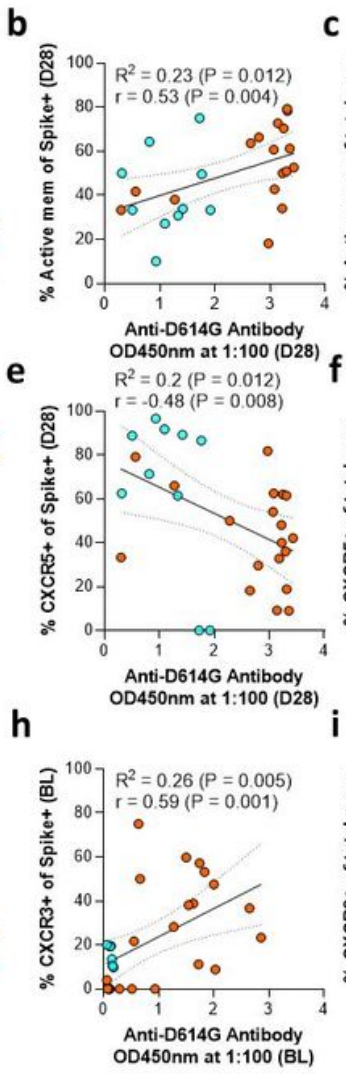

e
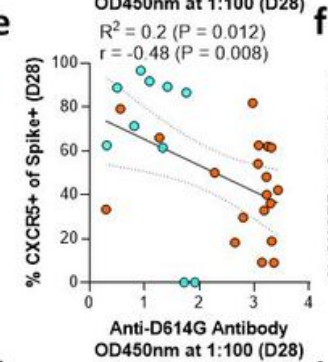

i

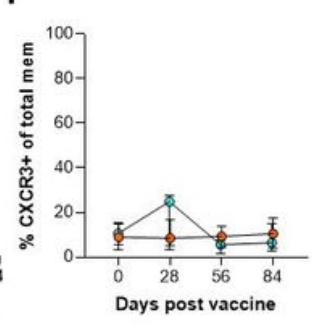

\section{Figure 3}

Longitudinal B cell activation and homing responses following vaccination. $a$, The Spike+ memory B cell population (blue) was overlaid onto the total B cell population (grey). Plotting CD27 against CD21 identifies a $C D 27+C D 21^{10}$ activated memory B cell population. Individual longitudinal plots for naïve (blue) and previously infected (orange) responses are followed by the median $\left({ }^{+} / .95 \% \mathrm{Cl}\right)$ longitudinal group responses. b, Spearman non-parametric correlation of the day 28 Spike+ active B cell memory versus the day 28 D614G spike-specific antibody response. c, The median total activated memory B cell responses were compared. The dashed lines represent the initial median response for the naïve (blue) and previously infected (orange) groups. A similar layout was followed in d-f, focusing on the CXCR5+ germinal center homing B cells and $\mathbf{g}-\mathbf{h}$, the lung homing CXCR3+ B cells. The correlation in $\mathbf{h}$ was of the baseline (BL) CXCR3+ and antibody response. Statistical analyses were performed using the Wilcoxon test for longitudinal time points versus day 0 and Mann-Whitney test between naïve and previously infected groups. $P$ values are denoted by $* \leq 0.05 ; * \star<0.01 ; * \star \star<0.001$ and $* \star \star \star<0.0001$. 
a
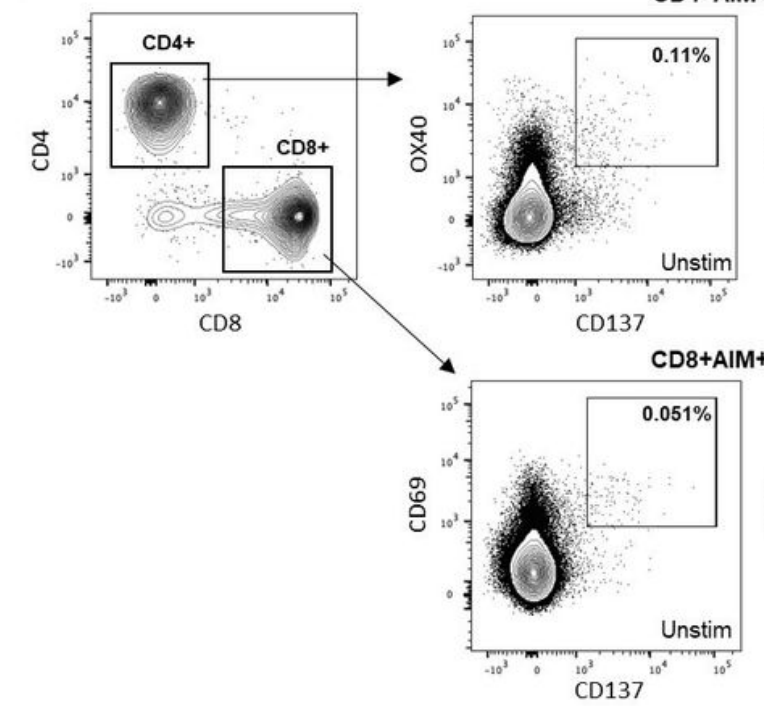

b

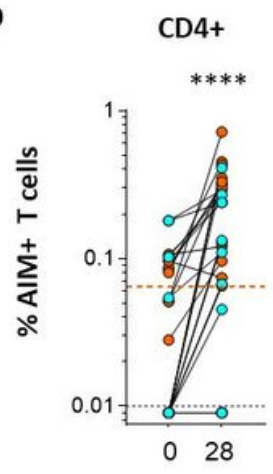

CD4+ cTfh

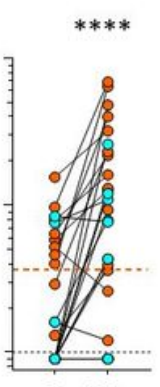

$0 \quad 28$

Days post vaccine
CD4+AIM+: OX40+CD137+

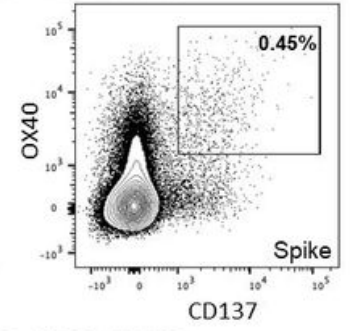

CD137

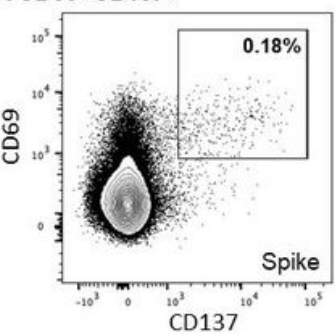

CD8+

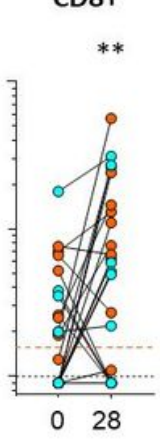

Naïve (10)
C

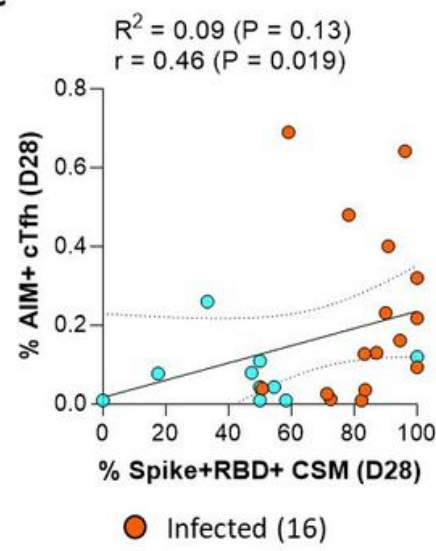

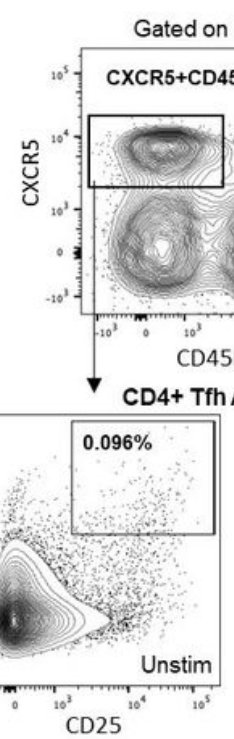

d

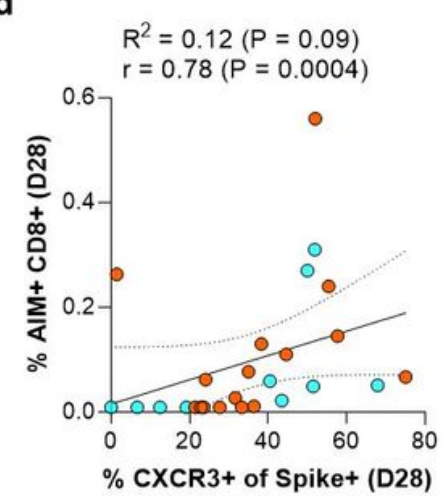

\section{Figure 4}

Spike specific T cell AIM responses prior to (baseline) and 28 days post-vaccination. a, Gating strategy identifying AIM+ T cells. Cells were first gated on live, CD3+ lymphocytes, and then total CD4+ or CD8+ T cells, with subsequent detection of CD4+AIM+ T cells (OX40+CD137+; top row) and CD8+AIM+ T cells (CD69+CD137+; bottom row). To identify cTfh cells, the CXCR5+CD45RA- CD4+ population was gated, with subsequent detection of AIM+ cells (OX40+CD25+). Representative plots for an unstimulated (Unstim) and Spike peptide pool-stimulated (Spike) sample are shown. Frequencies of Spike-specific AIM+ cells are indicated in the gates. $\mathbf{b}$, Total Spike-specific CD4+, CD4+ CTfh and CD8+ frequencies were compared prior to (baseline) and 28 days after vaccination, in naïve (blue) and previously infected (orange) groups. The dashed lines represent assay cut-off (black) and initial median response for the previously infected (orange) group. The starting median for the naïve group fell on/below the assay cutoff. c, Association between the frequency of AIM+cTfh cells and Spike+ RBD+ CSM B cells at day 28. d, Correlation between AIM+CD8+ cells and CXCR3+ Spike+ memory B cells at day 28. Sample numbers are show in brackets in the key. Statistical comparisons were performed using the Wilcoxon test for day 0 and day 28 time points. $P$ values are denoted by $* \star<0.01$ and $* \star \star \star<<0.0001$. Correlations were performed using the Spearman rank test. 
a

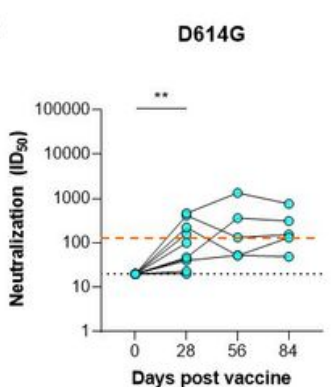

c

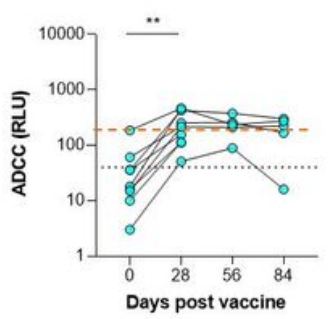

D614G

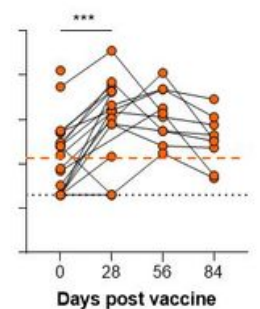

Days post vaccine

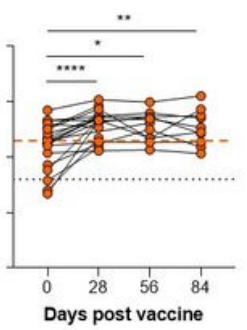

D614G

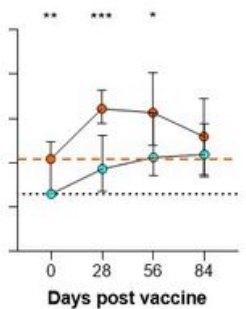

Days post vaccine

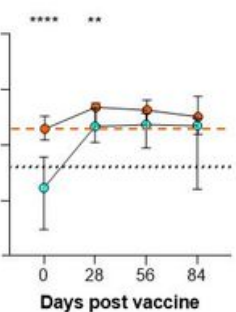

Naïve (O) b

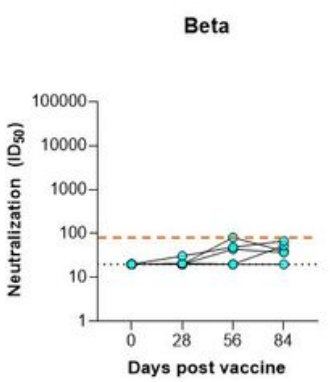

d
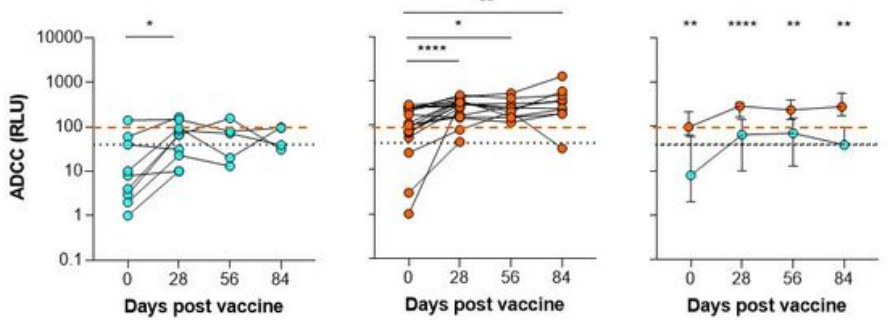

Infected (O)
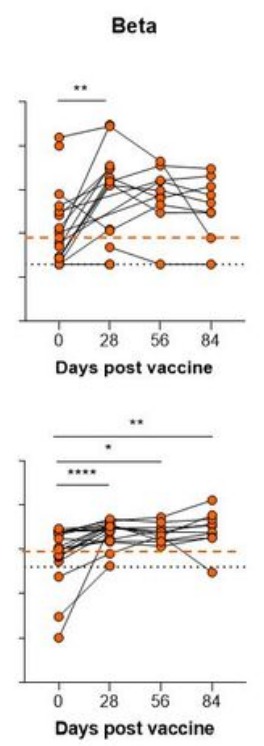

Days post vaccine
Days post vaccine

\section{Figure 5}

Longitudinal antibody dependent viral neutralization and cellular cytotoxicity. a,b, Viral neutralization $\mathrm{ID}_{50}$ titers versus the D614G or Beta variant respectively. Individual longitudinal plots are followed by a median $(+/ .95 \% \mathrm{Cl})$ plot for comparison between naïve (blue) and previously infected (orange) groups. c,d, Antibody dependent cellular cytotoxicity (ADCC) represented as relative light units for participant plasma's respective cross-linking of D614G or Beta spike-expressing cells and FcyRIIla. The dashed lines represent assay cut-off (black) and initial median response for the previously infected (orange) group. The starting median for the naïve group fell on/below the assay cut-off. Statistical analyses were performed using the Wilcoxon test for longitudinal time points versus day 0 and Mann-Whitney test between naïve and previously infected groups. $P$ values are denoted by $* \leq 0.05 ; * \star<0.01 ; * \star *<0.001$ and $* \star \star *<0.0001$. 


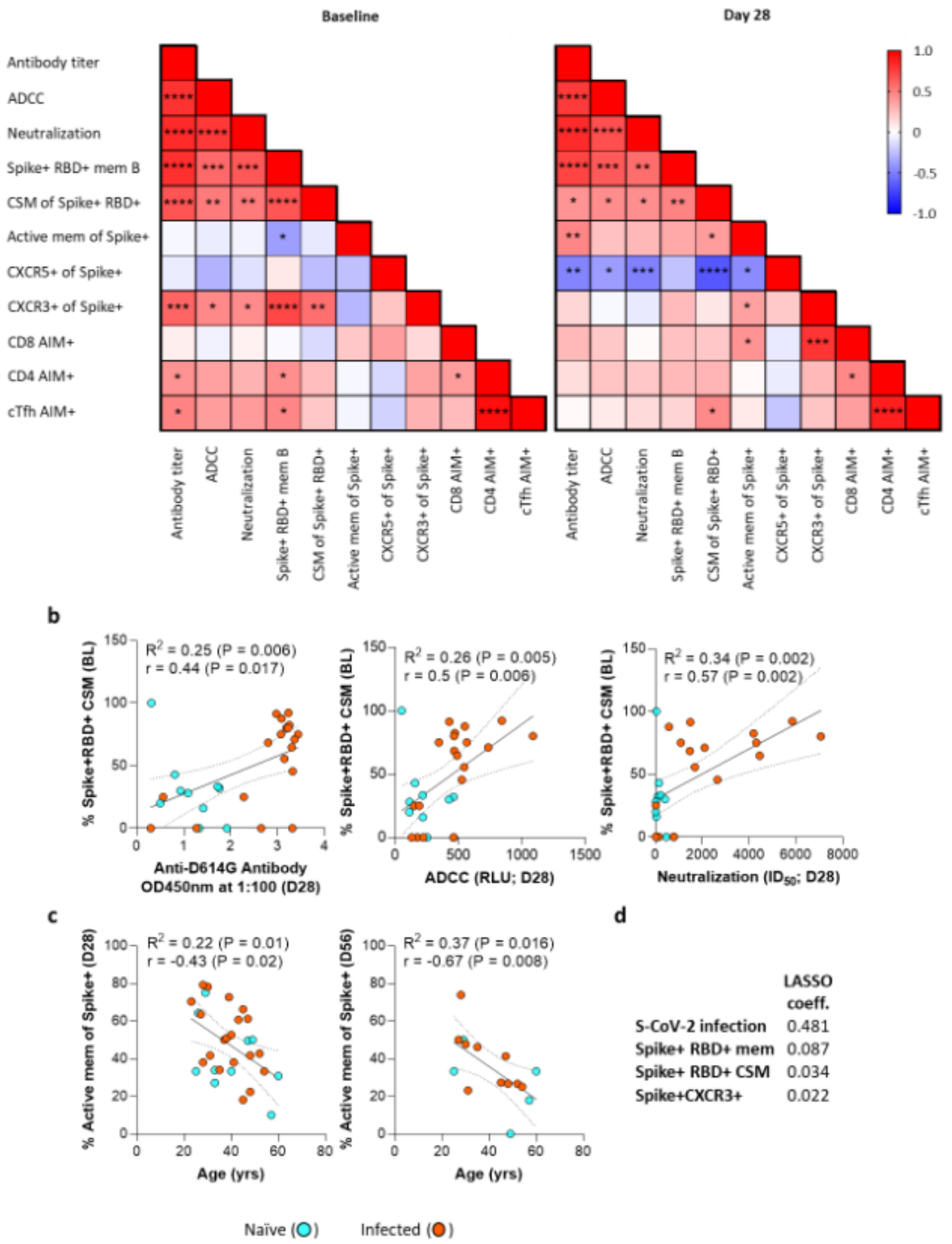

Figure 6

Correlations of the antibody, B and T cell responses following vaccination and the effect of age on B cell activation. a, The Spearman rank correlation values ( $r$ ) per comparison are presented as red (1.0) to blue (-1.0). b, Baseline (BL) class switched memory (CSM) and day 28 spike specific antibody, or day 28 ADCC, or day 28 neutralization were correlated. c, The extent of Spike+ memory B cell activation was correlated with age for all participants. The respective $P$ values denoted by $* \leq 0.05 ; * \star<0.01 ; * \star \star<0.001$ and $* \star \star \star<$ 
0.0001. Antibody dependent SARS-CoV-2 neutralization was selected as an outcome for LASSO regression analysis, with Spike+ memory B cell characteristics as covariates. The selected memory B cell predictors and their LASSO coefficient estimates were listed (intercept estimate was 4.338) in d.

\section{Supplementary Files}

This is a list of supplementary files associated with this preprint. Click to download.

- ExtendedData.docx 